Arq. Bras. Med. Vet. Zootec., v.56, n.3, p.385-391, 2004

\title{
Análise de custos de diferentes grupos genéticos de bovinos de corte terminados em confinamento
}

\author{
[Cost analyses on different beef cattle genetic groups finished in feedlot condition] \\ I.C. Ferreira ${ }^{1}$, M.A. Silva ${ }^{2}$, R.P. Reis ${ }^{3}$, K. Euclides Filho ${ }^{4}$, G.R. Figueiredo ${ }^{4}$ \\ ${ }^{1}$ Médica Veterinária, Mestre em Zootecnia - Escola de Veterinária da UFMG \\ ${ }^{2}$ Departamento de Zootecnia da Escola de Veterinária - UFMG \\ Caixa Postal 567 \\ 30123-970 - Belo Horizonte, MG \\ ${ }^{3}$ Departamento de Administração e Economia - UFLA - Lavras, MG \\ ${ }^{4}$ Embrapa Gado de Corte - Campo Grande, MS
}

\begin{abstract}
RESUMO
O desempenho de 149 animais, machos inteiros, confinados por períodos variáveis de acordo com acabamento de carcaça, foi utilizado para avaliar a eficiência econômica dos grupos genéticos Nelore sobreano, Nelore desmamado, $1 / 2$ Valdostana $1 / 2$ Nelore, $1 / 2$ Simental $1 / 2$ Nelore, $1 / 2$ Braford $1 / 2$ Brangus, $1 / 2$ Braford $1 / 4$ Angus $1 / 4$ Nelore, Brangus, $1 / 2$ Canchim $1 / 4$ Angus $1 / 4$ Nelore e $1 / 2$ Canchim $1 / 4$ Simental $1 / 4$ Nelore. O custo operacional variável, o custo por quilograma de carne produzida e a margem bruta foram analisados pelo método dos quadrados mínimos ordinários, mediante modelo estatístico que incluiu o efeito do grupo genético e o peso inicial como covariável, comparando-se as médias pelo teste Duncan $(\mathrm{P}<0,05)$. Os maiores custos operacionais variáveis no período, em US\$, foram do $1 / 2$ Canchim $1 / 4$ Simental 1/4 Nelore (160,8), Nelore desmamado (158,7) e 1/2 Canchim 1/4 Angus 1/4 Nelore $(155,1)$. Os menores custos por quilograma de carne, em US\$, foram do Nelore sobreano $(0,79)$ e Brangus $(0,82)$. A maior margem bruta, em US\$, foi obtida pelo grupo genético Nelore sobreano $(94,6)$, seguida do Brangus $(71,5)$ e do $1 / 2$ Simental $1 / 2$ Nelore $(46,6)$. Os grupos genéticos mais pesados no início do confinamento e que permaneceram menos tempo confinados apresentaram maior margem bruta.
\end{abstract}

Palavras- chave: bovino, grupo genético, confinamento, custo, margem bruta

\begin{abstract}
Data on feedlot performance of 149 steers were used to evaluate the economic efficiency of yearling Nellore, weaned Nellore, 1/2 Valdostana 1/2 Nellore, 1/2 Simmental 1/2 Nellore, 1/2 Braford 1/2 Brangus, $1 / 2$ Braford 1/4 Angus 1/4 Nellore, Brangus, 1/2 Canchim 1/4 Angus 1/4 Nellore and 1/2 Canchim 1/4 Simmental 1/4 Nellore. Variable operational costs, $\mathrm{kg}$ of meat costs, and gross margin income were analyzed by ordinary least squares procedure including the effects of genetic groups and initial weight in the feedlot as a covariate. The means were compared by Duncan test $(P<.05)$. The largest variable operational costs in the period, in US\$, were observed for 1/2 Canchim 1/4 Simmental 1/4 Nellore (160.8), weaned Nellore (158.7) and 1/2 Canchim 1/4 Angus 1/4 Nellore (155.1). The lowest $\mathrm{kg}$ of meat costs, in US\$, were observed for yearling Nellore (0.79) and Brangus (0.82). The highest gross margin was obtained for yearling Nellore (94.6), followed by Brangus (71.5) and $1 / 2$ Simmental $1 / 2$ Nellore (46.6) The heaviest genetic groups in the beginning of the feed lot were confined for shorter periods and had the largest gross margins.
\end{abstract}

Keywords: cattle, genetic group, feedlot, cost, gross margin

Recebido para publicação em 29 de abril de 2003

Recebido para publicação, após modificações, em 8 de fevereiro de 2004

E-mail: martinho@vet.ufmg.br 


\section{INTRODUÇÃO}

O Brasil tem alto potencial para produção de carne bovina em razão da extensão territorial e disponibilidade de pastagem. Contudo, os desempenhos técnico e econômico, geralmente, não asseguram alta rentabilidade pelos baixos índices de produtividade. Vários fatores, entre os quais a estacionalidade da produção de forragens, reduzem os ganhos alcançados na época das águas. O confinamento pode ser uma opção para reduzir os efeitos negativos do período seco.

Os indicadores econômicos são baseados na fundamentação teórica de custos de produção, sendo estimados por meio dos conceitos de custos operacionais variáveis e margem bruta. Os custos operacionais variáveis representam somente os desembolsos diretos com o confinamento. A margem bruta é a receita total menos os custos operacionais variáveis totais. Para obter a margem líquida, subtrai-se da receita total os custos operacionais variáveis totais, as depreciações dos recursos fixos e o custo alternativo de aplicação do capital empatado na atividade produtiva (Reis, 2002).

Cezar e Euclides Filho (1996) avaliaram a eficiência econômica do sistema de produção com novilho precoce e utilizaram a margem bruta como indicador econômico. Concluíram que o aumento de $30 \mathrm{~kg}$ no peso à desmama pode incrementar a margem bruta/ha/ano em $11 \%$ e a margem bruta/kg de carcaça em $12 \%$. Potter et al. (2000) realizaram análise econômica de modelos de produção com novilhas de corte primíparas aos dois, três e quatro anos de idade e concluíram que o sistema que proporcionou maior margem bruta foi o de dois anos.

Esta pesquisa teve por objetivo realizar análises de custos do confinamento de diferentes grupos genéticos, considerando a margem bruta como indicador de eficiência econômica, com vistas a determinar os grupos genéticos economicamente mais viáveis.

\section{MATERIAL E MÉTODOS}

Os dados foram provenientes de experimento realizado na EMBRAPA Gado de Corte - Campo Grande- MS, no período de julho a dezembro de 2000, e referem-se ao desempenho de 149 animais machos inteiros, confinados por períodos variáveis, de acordo com acabamento de carcaça, de nove diferentes grupos genéticos. Os animais foram oriundos do rebanho da EMBRAPA e de produtores da região que trabalham em sistemas de parceria, porém com diferentes sistemas de manejo animal. Os grupos genéticos apresentavam idades e pesos distintos no início do confinamento (Tab. 1). Os animais foram alimentados com volumoso (silagem de milho) e concentrado, na proporção de 50:50 da MS total da dieta.

O momento de abate foi definido por avaliação visual da cobertura de gordura na carcaça.

Tabela 1. Número de animais, idade e peso médios iniciais, com respectivos desvios padrão, por grupo genético

\begin{tabular}{|c|c|c|c|}
\hline Grupo genético & $\begin{array}{c}\text { Número } \\
\text { de animais }\end{array}$ & $\begin{array}{l}\text { Idade inicial média } \\
\text { (meses) }\end{array}$ & $\begin{array}{c}\text { Peso inicial médio } \\
(\mathrm{kg})\end{array}$ \\
\hline Nelore desmamado & 12 & $10,80 \pm 0,00$ & $258,42 \pm 18,00$ \\
\hline Nelore sobreano & 39 & $21,92 \pm 0,59$ & $394,13 \pm 22,17$ \\
\hline $1 / 2$ Valdostana $1 / 2$ Nelore & 22 & $19,77 \pm 0,89$ & $306,09 \pm 24,65$ \\
\hline $1 / 2$ Simental $1 / 2$ Nelore & 12 & $19,25 \pm 0,55$ & $336,92 \pm 44,54$ \\
\hline $1 / 2$ Braford $1 / 2$ Brangus & 12 & - & $267,75 \pm 20,97$ \\
\hline $1 / 2$ Braford $1 / 4$ Angus $1 / 4$ Nelore & 12 & $13,24 \pm 2,46$ & $257,33 \pm 21,44$ \\
\hline Brangus & 12 & $20,48 \pm 1,35$ & $390,58 \pm 48,47$ \\
\hline $1 / 2$ Canchim $1 / 4$ Angus $1 / 4$ Nelore & 12 & $8,13 \pm 0,55$ & $239,50 \pm 18,23$ \\
\hline $1 / 2$ Canchim $1 / 4$ Simental $1 / 4$ Nelore & 16 & $8,03 \pm 0,28$ & $236,50 \pm 17,53$ \\
\hline
\end{tabular}

O custo da ração para os grupos genéticos de animais de 8 a 13 meses foi de 131,53 US\$/ tonelada e para os animais de 19 a 22 meses foi de 106,87 US\$/tonelada. Nos últimos cinco anos (1998 - 2002), os preços médios do milho e da soja foram, respectivamente, 78,92 US\$/tonelada e 175,73 US\$/tonelada. 
Foram considerados como custos variáveis de cada grupo genético os gastos realizados com alimentação (volumosos e concentrados), mistura mineral, medicamentos, mão-de-obra mais encargos, outros gastos com mecanização, manutenção de equipamentos, máquinas, benfeitorias em geral, administração, taxas de energia elétrica e combustíveis.

O custo da silagem de milho foi obtido em junho de 2002, na Embrapa Gado de Corte, com produtividade média estimada de 35 toneladas de milho/hectare. Os custos incluíam as despesas realizadas com aquisição de calcário, adubação de plantio e cobertura, herbicidas, sementes, serviços mecânicos e confecção da silagem, totalizando 12,68 US\$/tonelada.

O custo do sal branco e do sal mineral foi de 199,22 US\$/tonelada, o de vermífugo e carrapaticida $(0,05 \mathrm{US} \$ / \mathrm{ml})$ e o de vacinas $(0,08$ US\$/dose). Foram obtidos no mercado de Campo Grande, MS, em abril de 2002, e fornecidos pela FNP consultoria (FMP..., 2002). Os gastos com mão-de-obra e encargos (0,05 US\$/animal/dia), mecanização ( 0,05 US\$/animal/dia), manutenção de benfeitorias $(0,02$ US\$/animal/dia $)$ e administração mais taxas (0,06 US\$/animal/dia) foram estimados de acordo com FMP Consultoria (FMP..., 2002).

Os custos com a compra de animais destinados ao confinamento foram obtidos no mercado de Campo Grande, MS, em junho/2002. De acordo com o peso médio aproximado dos animais do experimento (Tab. 2), o preço da arroba que compôs a receita com a venda dos animais confinados foi de 14,83 US $\$$ kg ou US\$ 0,99 US $\$ / \mathrm{kg}$; o preço médio aritmético da arroba do período de 1997 a 2002 foi de 19,18 US\$/kg ou 1,27 US\$/kg e desvio-padrão de US\$2,78/@, segundo FNP consultoria (FNP..., 2002).

Tabela 2. Preços de compra de animais magros no mercado de campo grande, ms, em junho de 2002 e média no período de 1997 a 2002

\begin{tabular}{lcc}
\hline $\begin{array}{l}\text { Peso } \\
(\mathrm{kg})\end{array}$ & $\begin{array}{c}\text { Preço (US\$) } \\
\text { (junho 2002) }\end{array}$ & $\begin{array}{c}\text { Preço médio (US\$) } \pm \text { desvio-padrão } \\
(1997-2002)\end{array}$ \\
\hline 235 & 154,0 & $173,04 \pm 24,83$ \\
285 & 164,0 & $191,20 \pm 28,53$ \\
320 & 182,0 & $213,01 \pm 31,65$ \\
380 & 191,0 & $228,87 \pm 35,64$ \\
\hline
\end{tabular}

As variáveis dependentes de desempenho (idade e peso de abate, duração do confinamento, ganho de peso, rendimento de carcaça e espessura de gordura) e os custos (custo operacional variável, custo por quilograma de carne produzida $\mathrm{e}$ margem bruta com receita de preços médios do quilograma de carne de boi no período de 1997 a 2002 e preços de junho de 2002) foram analisadas pelo método dos quadrados mínimos ordinários, de acordo com o seguinte modelo:

$\mathrm{Y}_{\mathrm{ij}}=+\mathrm{G}_{\mathrm{i}}+\mathrm{bX}_{\mathrm{ij}}+\mathrm{e}_{\mathrm{ij}}$, em que:

$\mathrm{Y}_{\mathrm{ij}}=$ variável dependente de cada animal $\mathrm{j}$ pertencente ao grupo genético $\mathrm{i}$;

$\alpha=$ constante;

$\mathrm{G}_{\mathrm{i}}=$ efeito do grupo genético $\mathrm{i}, \mathrm{i}=1,2 \ldots 9$;

$b=$ coeficiente de regressão da variável $Y_{i j}$ em relação ao peso do animal no início do confinamento;

$\mathrm{X}_{\mathrm{ij}}=$ peso do animal $\mathrm{j}$ no início do confinamento pertencente ao grupo genético $\mathrm{i}$;

$\mathrm{e}_{\mathrm{ij}}=$ erro associado a cada observação;

Os grupos genéticos para i (i variando de 1 a 9) foram: 1= Nelore desmamado, 2= Nelore sobreano, $3=1 / 2$ Valdostana $1 / 2$ Nelore, $4=1 / 2$ Simental $1 / 2$ Nelore, 5=1/2 Braford $1 / 2$ Brangus, $6=$ $1 / 2$ Braford $1 / 4$ Angus $1 / 4$ Nelore, $7=$ Brangus, $8=1 / 2$ Canchim $1 / 4$ Angus $1 / 4$ Nelore e 9= $1 / 2$ Canchim $1 / 4$ Simental $1 / 4$ Nelore.

As médias dos grupos genéticos foram comparadas pelo teste Duncan, até o nível de 5\% de probabilidade.

O modelo analítico utilizado para estimar o custo operacional variável para cada animal, pertencente a cada grupo genético, foi definido pela expressão:

$\operatorname{CopV}_{\mathrm{ij}}=\sum_{\mathrm{m}=1}^{5}\left(\mathrm{Px}_{\mathrm{ij}} \mathrm{X}_{\mathrm{i}}\right)+\sum_{\mathrm{n}=1}^{5}\left(\mathrm{~K}_{\mathrm{ij}}\right) \mathrm{n}$, em que:

$\operatorname{CopV}_{\mathrm{ij}}=$ custo operacional variável de cada animal $\mathrm{j}$, pertencente ao grupo genético $\mathrm{i}$;

$\left(\mathrm{Px}_{\mathrm{ij}}\right) \mathrm{m}=$ preço do concentrado $(\mathrm{m}=1)$, volumoso $(\mathrm{m}=2)$, mistura mineral $(\mathrm{m}=3)$, vermífugo $(\mathrm{m}=4)$ e carrapaticida $(\mathrm{m}=5)$ para cada animal $\mathrm{j}$, pertencente ao grupo genético $i$;

$\mathrm{X}_{\mathrm{i}}=$ quantidade de cada item utilizado pelo grupo genético $i$, para $i=1,2 \ldots 9$;

$\left(\mathrm{K}_{\mathrm{ij}}\right) \mathrm{n}=$ custos dos fatores mantidos constantes para cada animal $\mathrm{j}$ pertencentes a todos os grupos genéticos $i$, vacina $(n=1)$, mão-de-obra mais encargos $(n=2)$, mecanização $(n=3)$, manutenção de benfeitorias $(n=4)$ e administração mais taxas $(n=5)$; 
A receita foi obtida considerando-se o peso de abate no frigorífico com rendimento médio de carcaça de $56 \%$. Essa receita foi estimada de acordo com o preço do quilograma de carne em junho de 2002, e com o preço médio do quilograma de carne no período de 1997 a 2002.

O modelo analítico utilizado para estimar a receita para cada animal, pertencente a cada grupo genético, foi definido pela expressão:

$\mathrm{R}_{\mathrm{ij}}=\mathrm{Pa}_{\mathrm{ij}} \mathrm{Xa}_{\mathrm{ij}}$, em que;

$\mathrm{R}_{\mathrm{ij}}=$ receita de cada animal $\mathrm{j}$ pertencente ao grupo genético $\mathrm{i}$;

$\mathrm{Pa}_{\mathrm{ij}}=$ preço do quilograma de carne de cada animal j pertencente ao grupo genético $\mathrm{i}$;

$\mathrm{Xa}_{\mathrm{ij}}=$ quilograma de carne produzido pelo animal $\mathrm{j}$ pertencente ao grupo genético $\mathrm{i}$; sendo $\mathrm{i}=1,2 \ldots 9$ e $\mathrm{j}=$ cada animal pertencente ao grupo genético $\mathrm{i}$.

O modelo analítico utilizado para estimar a margem bruta de cada animal, pertencente a cada grupo genético, foi expressado como:
$\mathrm{MB}_{\mathrm{ij}}=\mathrm{R}_{\mathrm{ij}}-\left(\mathrm{CopV}_{\mathrm{ij}}+\mathrm{CA}_{\mathrm{ij}}\right)$, em que:

$\mathrm{MB}_{\mathrm{ij}}=$ margem bruta do animal j pertencente ao grupo genético i;

$\mathrm{R}_{\mathrm{ij}}=$ receita de cada animal $\mathrm{j}$ pertencente ao grupo genético $\mathrm{i}$;

$\operatorname{CopV}_{\mathrm{ij}}=$ custo operacional variável de cada animal j pertencente ao grupo genético $\mathrm{i}$;

$\mathrm{CA}_{\mathrm{ij}}=$ custo do animal magro $\mathrm{j}$ no início do experimento, pertencente ao grupo genético $\mathrm{i}$;

sendo $\mathrm{i}=1,2 \ldots 9$ e $\mathrm{j}=$ cada animal pertencente ao grupo genético $\mathrm{i}$.

\section{RESULTADOS E DISCUSSÃO}

Os resultados de desempenho dos nove grupos genéticos são apresentados na Tab. 3. Salienta-se que os grupos genéticos apresentavam diferenças quanto à origem, manejo pré confinamento, idade inicial de entrada no confinamento e espaço nos piquetes.

Tabela 3. Idade e peso ao abate, duração e ganho diário médio (GDM) de acordo com o grupo genético de bovinos em confinamento

\begin{tabular}{|c|c|c|c|c|}
\hline Grupo genético & $\begin{array}{c}\text { Idade de abate } \\
\text { (meses) }\end{array}$ & $\begin{array}{c}\text { Duração do } \\
\text { confinamento (dias) }\end{array}$ & $\begin{array}{c}\text { Peso ao } \\
\text { abate }(\mathrm{kg})\end{array}$ & $\begin{array}{c}\text { GDM } \\
(\mathrm{kg} / \mathrm{dia})\end{array}$ \\
\hline Nelore desmamado & $16,40 \mathrm{~d}$ & $168,00 \mathrm{a}$ & $481,83 \mathrm{~cd}$ & $1,32 \mathrm{c}$ \\
\hline Nelore sobreano & $23,66 b$ & $52,10 \mathrm{e}$ & $452,05 \mathrm{e}$ & $1,62 \mathrm{ab}$ \\
\hline $1 / 2$ Valdostana $1 / 2$ Nelore & $24,83 \mathrm{a}$ & $151,50 \mathrm{~b}$ & $513,52 \mathrm{a}$ & $1,34 \mathrm{c}$ \\
\hline $1 / 2$ Simental $1 / 2$ Nelore & $23,09 b$ & $113,80 \mathrm{c}$ & $526,92 \mathrm{a}$ & $1,70 \mathrm{a}$ \\
\hline $1 / 2$ Braford $1 / 2$ Brangus & - & $148,70 \mathrm{~b}$ & $490,59 \mathrm{bc}$ & $1,49 \mathrm{abc}$ \\
\hline $1 / 2$ Braford $1 / 4$ Angus $1 / 4$ Nelore & $18,43 \mathrm{c}$ & $155,50 \mathrm{~b}$ & $483,64 \mathrm{~cd}$ & $1,45 \mathrm{bc}$ \\
\hline Brangus & $22,92 b$ & $62,40 \mathrm{~d}$ & $486,70 \mathrm{bcd}$ & $1,66 a b$ \\
\hline $1 / 2$ Canchim $1 / 4$ Angus $1 / 4$ Nelore & $13,84 \mathrm{e}$ & $168,00 \mathrm{a}$ & $464,25 \mathrm{de}$ & $1,33 \mathrm{c}$ \\
\hline $1 / 2$ Canchim $1 / 4$ Simental $1 / 4$ Nelore & $13,56 \mathrm{e}$ & $166,40 \mathrm{a}$ & $508,81 \mathrm{ab}$ & $1,63 \mathrm{ab}$ \\
\hline
\end{tabular}

Médias seguidas de letras distintas, na coluna, diferem entre si pelo teste Duncan $(\mathrm{P}<0,0001)$.

Os animais que imediatamente após o desmame seguiram para o confinamento e foram abatidos antes dos 15 meses de idade foram considerados superprecoces. Os que passaram por um período de recria, confinados e abatidos até 24 meses de idade foram considerados precoces.

A duração do confinamento foi maior para os grupos Nelore desmamado, $1 / 2$ Canchim $1 / 4$ Angus $1 / 4$ Nelore e $1 / 2$ Canchim $1 / 4$ Simental $1 / 4$ Nelore; Tab. 3. Segundo Cardoso (2000) e Restle et al. (2000), os animais jovens que ainda estão em fase de crescimento, necessitam de mais tempo para direcionar energia aos crescimentos ósseo, muscular e acabamento.
No conjunto dos precoces, o Nelore sobreano apresentou o menor período de confinamento, seguido do Brangus e do $1 / 2$ Simental $1 / 2$ Nelore. Segundo Cardoso (2000) e Restle et al. (2000), os animais com maior peso inicial, isto é, aqueles que no período de confinamento apenas depositam musculo e gordura, não precisam de direcionamento energético para crescimento ósseo como os de menor peso inicial.

Segundo Barbosa (1998), o peso de abate foi $11 \%$ superior para os animais cruzados em relação aos puros. Nesta pesquisa verificou-se superioridade dos cruzados em relação aos puros somente para os grupos genéticos $1 / 2$ Simental $1 / 2$ 
Nelore, $1 / 2$ Valdostana $1 / 2$ Nelore e $1 / 2$ Canchim $1 / 4$ Simental $1 / 4$ Nelore, apesar de a idade inicial e as condições pré-confinamento não serem semelhantes. O maior peso desses grupos deveuse, provavelmente, à composição do grupo genético, com participação de raças de grande porte. De acordo com Peixoto (1996), Vaz et al. (1999), Cardoso (2000) e Restle et al. (2000), as raças Simental e Canchim, por serem de grande porte e mais tardias na deposição de gordura, atrasam o acabamento e favorecem o maior peso ao abate.

As rações foram balanceadas para ganho de peso de $1,250 \mathrm{~kg} / \mathrm{dia}$. No entanto, todos os grupos genéticos o superaram (Tab. 3), provavelmente em função do ganho compensatório, pois as condições nutricionais pré-confinamento não foram controladas.

Os maiores ganhos diários médios foram para os grupos $1 / 2$ Simental $1 / 2$ Nelore, Brangus, 1/2 Canchim $1 / 4$ Simental $1 / 4$ Nelore, Nelore sobreano e $1 / 2$ Braford $1 / 2$ Brangus. Não se observou diferença entre esses grupos, mesmo sabendo que não havia uniformidade de manejo no préconfinamento. Barbosa (1998) verificou superioridade de até $20 \%$ em animais cruzados quando comparados aos puros.

Quanto ao rendimento de carcaça (Tab. 4), os maiores valores foram observados nos grupos genéticos Nelore desmamado $(60,0 \%)$ e $1 / 2$ Braford $1 / 4$ Angus $1 / 4$ Nelore (59,3\%), que diferiram dos grupos genéticos $1 / 2$ Simental $1 / 2$ Nelore $(57,7 \%)$ e Brangus $(57,4 \%)$. Estes dois últimos grupos apresentaram rendimento de carcaça semelhante ao do $1 / 2$ Valdostana $1 / 2$ Nelore $(58,6 \%)$ e do $1 / 2$ Braford $1 / 2$ Brangus $(58,6 \%)$. O maior rendimento de carcaça do grupo genético Nelore confirma o estudo de Restle et al. (1999), segundo o qual, zebuínos apresentam maior rendimento de carcaça do que animais de origem européia e seus cruzamentos, graças ao menor peso relativo das patas, cabeça, couro e trato digestivo.

Como o grau de acabamento foi a característica definida para abate dos animais, esperava-se que não houvesse diferença significativa entre os grupos genéticos para espessura de gordura. Entretanto, $1 / 2$ Braford $1 / 2$ Brangus e $1 / 2$ Braford $1 / 4$ Angus $1 / 4$ Nelore apresentaram maior espessura de gordura quando comparados aos demais grupos. A avaliação do grau de acabamento foi visual, menos precisa do que o método do ultra som (Hassen et al., 1999).

Tabela 4. Rendimento de carcaça e espessura de gordura de acordo com o grupo genético de bovinos em confinamento

\begin{tabular}{lcc}
\hline Grupo genético & $\begin{array}{c}\text { Rendimento de } \\
\text { carcaça }(\%)\end{array}$ & $\begin{array}{c}\text { Espessura de } \\
\text { gordura }(\mathrm{mm})\end{array}$ \\
\hline Nelore desmamado & $59,97 \mathrm{a}$ & $4,75 \mathrm{~b}$ \\
$1 / 2$ Valdostana $1 / 2$ Nelore & $58,56 \mathrm{bc}$ & $4,27 \mathrm{~b}$ \\
$1 / 2$ Simental $1 / 2$ Nelore & $57,69 \mathrm{c}$ & $4,85 \mathrm{~b}$ \\
$1 / 2$ Braford $1 / 2$ Brangus & $58,61 \mathrm{bc}$ & $7,41 \mathrm{a}$ \\
$1 / 2$ Braford $1 / 4$ Angus $1 / 4$ Nelore & $59,32 \mathrm{ab}$ & $6,9 \mathrm{a}$ \\
Brangus & $57,45 \mathrm{c}$ & $4,3 \mathrm{~b}$ \\
\hline
\end{tabular}

Médias seguidas de letras distintas na coluna diferem entre si pelo teste Duncan $(\mathrm{P}<0,003$ para rendimento de carcaça e $\mathrm{P}<0,0003$ para espessura de gordura)

O concentrado foi o item de maior contribuição para o custo operacional variável total (CopVT), variando de 58,9 a $64,6 \%$. A alimentação representou de 80,2 a $83,9 \%$ do CopVT. Ainda, 4,8 a $5,9 \%$ referiram-se à mão-de-obra, 4,1 a $5,1 \%$ à mecanização, 1,4 a $1,8 \%$ à manutenção e 5,5 a $6,8 \%$ à administração e taxas. Segundo Muir et al. (1995), no confinamento $80 \%$ dos custos foram atribuídos à alimentação.

O menor custo operacional variável médio (CopVMe) por quilograma de carne produzido foi o do grupo genético Nelore sobreano, seguido do Brangus (Tab. 5), ambos diferindo dos demais grupos genéticos.

Entre os superprecoces, o grupo genético $1 / 2$ Canchim $1 / 4$ Simental $1 / 4$ Nelore apresentou 0 menor CopVMe por quilograma de carne produzido, quando comparado ao $1 / 2$ Canchim $1 / 4$ Angus $1 / 4$ Nelore, diferença atribuída ao maior ganho médio diário do primeiro grupo. Esse mesmo grupo diferiu dos grupos $1 / 2$ Valdostana $1 / 2$ Nelore e Nelore desmamado. Os demais grupos foram semelhantes entre si quanto ao CopVMe por quilograma de carne produzido.

Os dados do CopVMe e os dos preços médios de venda da carne em junho de 2002 (US\$0,99) permitiram a análise de rentabilidade simplificada do confinamento em função do grupo genético. Em razão do preço do quilograma de carne ter sido superior ao do CopVMe dos grupos genéticos Nelore sobreano, Brangus e $1 / 2$ Canchim $1 / 4$ Simental $1 / 4$ Nelore, para esses grupos o confinamento cobriu $o$ 
desembolso imediato, ou seja, o capital de giro, o que, segundo Reis (2002), indica uma situação em que a atividade produtiva pode ser mantida a curto prazo. No entanto, essa análise fica limitada, pois não inclui o custo operacional fixo, ou seja, a depreciação dos recursos fixos.

Tabela 5. Custo operacional variável total (CopVT), custo operacional variável médio (CopVMe) por quilograma de carne produzido e margem bruta (MB) segundo grupo genético de bovinos em confinamento

\begin{tabular}{lcccc}
\hline Grupo genético & $\begin{array}{c}\text { CopVT } \\
\text { US\$/período }\end{array}$ & $\begin{array}{c}\text { CopVMe } \\
\text { (US } \$ / k g)\end{array}$ & $\begin{array}{c}\mathrm{MB}^{1} \\
\text { junho/2002 }\end{array}$ & $\begin{array}{c}\mathrm{MB}^{2} \\
\text { preços médios }\end{array}$ \\
\hline Nelore desmamado & $158,67 \mathrm{a}$ & $1,12 \mathrm{a}$ & $-66,62 \mathrm{e}$ & $6,38 \mathrm{e}$ \\
Nelore sobreano & $54,35 \mathrm{~d}$ & $0,79 \mathrm{c}$ & $16,74 \mathrm{a}$ & $94,56 \mathrm{a}$ \\
$1 / 2$ Valdostana $1 / 2$ Nelore & $142,33 \mathrm{~b}$ & $1,11 \mathrm{a}$ & $-48,55 \mathrm{~d}$ & $28,93 \mathrm{~d}$ \\
$1 / 2$ Simenta1 $1 / 2$ Nelore & $130,21 \mathrm{c}$ & $1,04 \mathrm{ab}$ & $-35,44 \mathrm{c}$ & $46,61 \mathrm{c}$ \\
$1 / 2$ Braford $1 / 2$ Brangus & $143,34 \mathrm{~b}$ & $1,02 \mathrm{ab}$ & $-54,67 \mathrm{de}$ & $20,06 \mathrm{de}$ \\
$1 / 2$ Braford $1 / 4$ Angus $1 / 4$ Nelore & $145,26 \mathrm{~b}$ & $1,03 \mathrm{ab}$ & $-53,08 \mathrm{~d}$ & $19,97 \mathrm{de}$ \\
Brangus & $63,16 \mathrm{~d}$ & $0,82 \mathrm{c}$ & $-3,04 \mathrm{~b}$ & $71,49 \mathrm{~b}$ \\
$1 / 2$ Canchim $1 / 4$ Angus $1 / 4$ Nelore & $155,15 \mathrm{a}$ & $1,11 \mathrm{a}$ & $-58,49 \mathrm{de}$ & $15,87 \mathrm{de}$ \\
$1 / 2$ Canchim $1 / 4$ Simental $1 / 4$ Nelore & $160,80 \mathrm{a}$ & $0,95 \mathrm{~b}$ & $-49,19 \mathrm{~d}$ & $29,57 \mathrm{~d}$ \\
\hline
\end{tabular}

Médias seguidas de letras distintas na coluna diferem pelo teste Duncan $(\mathrm{P}<0,0001)$.

${ }^{1}$ Margem bruta com a receita de preços da carne de boi de junho de 2002

${ }^{2}$ Margem bruta com a receita de preços médios da carne de boi no período de 1997 a 2002.

Em relação aos demais grupos genéticos, o preço do quilograma de carne (US\$ 0,99 ) foi inferior ao do CopVMe (Tab. 5). Isto indica, segundo Reis (2002), que o confinamento teria continuidade somente se os custos variáveis fossem cobertos por recursos provenientes de outras atividades produtivas, ou seja, uma atividade subsidiaria outra dentro da propriedade.

No entanto, quando se compara CopVMe ao preço médio do quilograma de carne de 1997 a 2002 (US\$ 1,27), verifica-se que todos os grupos genéticos são capazes de cobrir os custos operacionais variáveis, indicando que o confinamento deles se sustenta pelo menos no curto prazo.

A margem bruta com receita de junho de 2002 foi positiva somente para o grupo genético Nelore sobreano; os demais apresentaram margem bruta negativa. Entretanto, a margem bruta obtida com preços médios entre 1997 a 2002 foi positiva para todos os grupos genéticos. As maiores margens brutas, estatisticamente diferentes entre si, foram apresentadas pelo Nelore sobreano (US\$ 94,56), Brangus (US\$ 71,49 ) e $1 / 2$ Simental $1 / 2$ Nelore (US\$ 46,61). A menor margem bruta foi do Nelore desmamado (US\$ 6,38). As margens brutas dos demais grupos genéticos $(1 / 2$ Braford $1 / 2$ Brangus, $1 / 2$ Braford $1 / 4$ Angus $1 / 4$ Nelore e $1 / 2$ Canchim $1 / 4$
Angus $1 / 4$ Nelore) não diferiram da margem bruta do $1 / 2$ Valdostana $1 / 2$ Nelore, do $1 / 2$ Canchim $1 / 4$ Simental $1 / 4$ Nelore e do Nelore desmamado (Tab. 5).

A margem bruta com preços de junho de 2002 foi desfavorável para todos os grupos genéticos, exceto para o Nelore sobreano. A margem bruta com preços médios entre 1997 a 2002 indicou a viabilidade, pelo menos a curto prazo, de todos os grupos genéticos.

Os grupos genéticos mais viáveis, nas condições experimentais e analíticas, por apresentarem maior margem bruta, foram o Nelore sobreano, Brangus e $1 / 2$ Simental $1 / 2$ Nelore (Tab. 5). Esses animais já haviam, quando confinados, passado pelo período de crescimento e, portanto, ficaram menos tempo no confinamento, quando houve deposição de proteína muscular e gordura na carcaça. Dos três grupos genéticos que se destacaram, o maior desempenho do Nelore sobreano foi, provavelmente, em razão de sua idade no início do confinamento (21,92 meses), seguido do Brangus (20,48 meses) e do $1 / 2$ Simental $1 / 2$ Nelore (19,52 meses). Assim, quanto mais rápido o animal passar pelo confinamento mais eficiente economicamente será o sistema, porque o custo com alimentação representa, aproximadamente, $80 \%$ do custo operacional variável total. 
Finalmente, salienta-se que a análise econômica foi feita apenas para o período de confinamento. Neste caso, o conjunto dos superprecoces foi menos eficiente economicamente, apesar do grupo genético $1 / 2$ Canchim $1 / 4$ Simental $1 / 4$ Nelore apresentar alto ganho diário médio. Isto indica que no confinamento de superprecoces deve-se utilizar grupos genéticos com alta capacidade de ganho de peso e eficiência de conversão de alimento em carne no menor tempo (Amer et al., 1994).

\section{CONCLUSÕES}

De acordo com os indicadores econômicos do confinamento, com o preço da carne a 0,99 US $\$ / \mathrm{kg}$, somente os grupos genéticos Nelore sobreano, Brangus e $1 / 2$ Canchim 1/4 Simental 1/4 Nelore conseguem cobrir o custo operacional variável. Entretanto, com o preço da carne a 1,27 US $\$ / \mathrm{kg}$ todos os grupos genéticos cobrem os custos operacionais variáveis. As maiores margens são obtidas para grupos genéticos que entram em confinamento mais pesados e permanecem menor tempo confinados. Considerando os preços da tonelada de soja (US\$ 173,8 ) em junho de 2002, de milho (US\$ 77,5) e da carne de boi $(0,99$ US $\$ / \mathrm{kg})$ em junho de 2002, o confinamento de todos os grupos genéticos apresenta margem bruta negativa, exceto o grupo Nelore sobreano. Nas condições de preços médios entre 1997 e 2002, com preços da tonelada de soja (US\$ 175,7), de milho (US\$ 78,9 ) e da carne de boi (US\$ $1,27 / \mathrm{kg}$ ), todos os grupos genéticos apresentam margem bruta positiva.

\section{REFERÊNCIAS BIBLIOGRÁFICAS}

AMER, P.R.; KEMP, R.A.; BUCHANAN-SMITH, J.C. et al. A bioeconomic model for comparing beef cattle genotypes at their optimal economic slaughter end point. J. Anim. Sci., v.72, p.38-50, 1994.

BARBOSA, P.F. Cruzamentos industriais e a produção de novilhos precoces. In: SIMPÓSIO SOBRE PRODUÇÃO INTENSIVA DE GADO DE CORTE, 1., 1998, Campinas. Anais... Campinas: CBNA, 1998. p.117-134.
CARDOSO, E.G.C. Confinamento de bovinos. In: CURSO DE SUPLEMENTAÇÃO EM PASTO E CONFINAMENTO DE BOVINOS, 2000, Campo Grande. Disponível em: <www.cnpgc. embrapa.br/publicações/nãoseriadas/cursosuplemen tação/confinamento> Acessado em: 17/12/2002.

CÉZAR, I.M.; EUCLIDES FILHO, K. Novilho precoce: reflexos na eficiência e economicidade do sistema de produção. Campo Grande: Embrapa, 1996. (Documento, n.66).

FNP consultoria e comércio. 2002. Disponível em: <www.fnp.com.br>. Acessado em: 09/12/2002.

HASSEN, A.; WILSON, D.E.; ROUSE, G.H. Evaluation of carcass, live, and real-time ultrasound measures in feedlot cattle: I.assessment of sex and breed effects. J. Anim. Sci., v.77, p.273-282, 1999.

MUIR, P.D.; SMITH, N.B.; WALLACE, G.J. The cost of producing feedlot in New Zealand. Proceed. New Zeal. Soc. Anim. Prod., v.55, p.255-256, 1995.

PEIXOTO, A.M. Bovinos para confinamento. In: PEIXOTO, A.M.; HADDAD, C.M.; BOIN, C. et al. $O$ Confinamento de bois. 5.ed. São Paulo: Globo, 1996. p.17-48.

POTTER, L.; LOBATO, J.F.P.; MIELITZ NETTO, C.G.A. Análises econômicas de modelos de produção com novilhas de corte primíparas aos dois, três e quatro anos de idade. Rev. Bras. Zootec., v.29, p.861-870, 2000.

REIS, R.P. Fundamentos de economia aplicada. Lavras: UFLA/FAEPE, 2002. 95p. (Curso de Pós Graduação "Latu Sensu" (Especialização a distância).

RESTLE, J.; ALVES FILHO, D.C.; NEUMANN, M. Eficiência na terminação de bovinos de corte. In: Eficiência na produção de bovinos de corte. Santa Maria: UFSM, 2000. p.277303.

RESTLE, J.; VAZ, F.N.; QUADROS, A.R.B. et al.. Características de carcaça e da carne de diferentes genótipos de Hereford X Nelore. Rev. Bras. Zootec., v.28, p.1245-1251, 1999.

VAZ, F.N.; VAZ, R.Z.; BERNARDES, R.A.C. Viabilidade econômica do confinamento no Rio Grande do Sul. In: RESTLE, J. (Coord.). Confinamento, pastagens e suplementação para produção de bovinos de corte. Santa Maria: UFSM, 1999. p.147-177. 